\title{
Nasal carriage of Staphylococcus aureus among healthcare workers in relation to patient contact
}

\author{
Hsin-I Shih \\ National Cheng Kung University Hospital \\ Fan-Ching Shen \\ National Health Research Institutes \\ Yun-Ju Lee \\ National Health Research Institutes \\ Hsiang-Chin Hsu \\ National Cheng Kung University Hospital \\ Chiu-Hui Wu \\ National Cheng Kung University Hospital \\ Chia-Ming Chang \\ National Cheng Kung University Hospital \\ Chia-Yu Chi ( $\sim$ pedchi@nhri.org.tw) \\ National Health Research Institutes https://orcid.org/0000-0003-4481-3102
}

\section{Research article}

Keywords: MRSA, long term care facilities, healthcare worker, emergency medical technician

Posted Date: April 15th, 2019

DOI: https://doi.org/10.21203/rs.2.9188/v1

License: (c) (i) This work is licensed under a Creative Commons Attribution 4.0 International License.

Read Full License 


\section{Abstract}

Background: Methicillin-resistant Staphylococcus aureus (MRSA) is a major public health concern worldwide. Healthcare workers (HCWs) are an important source of transmission of MRSA. We conducted a prospective study to define the frequency of MRSA nasal colonization in HCWs in relation to the intensity of patient contact. Methods: Prevalence of MRSA and methicillin-susceptible S. aureus (MSSA) nasal colonization in HCWs was compared. These included an emergency department, intensive care unit, out-of-hospital care emergency medical technicians and students, and a long-term care facility (LTCF). The MRSA isolates were further identified by their microbiological and molecular characteristics. Results: S. aureus was isolated from 63 of $248 \mathrm{HCWs} \mathrm{(25.4 \% ).} \mathrm{The} \mathrm{overall} \mathrm{MRSA} \mathrm{nasal} \mathrm{carriage} \mathrm{rate} \mathrm{was}$ $15 / 248,6 \%$. Most MRSA carriers were female (14/15, 93.3\%), and HCWs who had worked for $\geq 5$ years $(11 / 15,73.3 \%)$. LTCFs had the highest prevalence $(3 / 25,12 \%)$. In contrast, the overall carriage of MSSA was $48 / 248,19.4 \%$, and most carriers worked for $\geq 5$ years $(25 / 48,52.1 \%)$. Hospital nurses had the highest rate of MSSA carriage (22/103, 21.4\%). Most of the MRSA isolates were SCCmec IV/ST59 or ST45 (60\%), and were resistant to erythromycin and clindamycin (53\%). Only one MRSA was chlorhexidine resistant. All produced low level of biofilms. Conclusions: This study demonstrates important differences in the characteristics of nasal carriage of MRSA and MSSA among HCWs. Inclusion of all strains of S. aureus in surveillance and infection control programs is warranted.

\section{Background}

Staphylococcus aureus is an important human pathogen. It has the ability to cause a wide variety of infections ranging from local invasion of skin and soft tissues to life-threatening sepsis. The emergence and spread of methicillin-resistant $S$. aureus (MRSA) is particularly troublesome because of its association with increased morbidity and mortality $[1,2]$ and the need to select the most appropriate therapy. Biofilm-forming variants are often difficult to treat even when susceptible to otherwise effective antibiotics.

MRSA infections are usually divided into healthcare-associated (HA-MRSA) or community-associated (CA-MRSA) because of differences in epidemiology, risk factors and choice of drug. HA-MRSA infections are more likely to occur in individuals with underlying diseases, the elderly, recent hospitalization, invasive procedures and residence in long term health care facilities [3]. CA-MRSA infections usually occur in otherwise healthy people and with minor trauma. HA-MRSA strains usually possess SCCmec types I, II or $\mathrm{III}$, and tend to be multiple drug resistant. CA-MRSA strains usually possess SCCmec types IV or V and are strongly associated with the Panton-Valentin leucocidin $(p v l)$ gene. CA-MRSA strains are considered to be more virulent, transmittable, and persistent than HA-MRSA [4, 5]. CA-MRSA strains can also be transmitted in healthcare facilities and mistaken for HA-MRSA [5].

The ability of $S$. aureus to colonize the anterior nares and other body sites is a significant predisposing risk factor for infection [5, 6]. Elimination of carriage decreases the incidence of $S$. aureus infection [7, 8]. The prevalence of MRSA nasal colonization in worldwide surveys of general populations ranges from 0.7 
to $3 \%[9,10]$. The rate is somewhat higher in Taiwan, $3.8 \%[11,12]$. Hospitalized patients and those in long-term care facilities (LTCF) are at highest risk for MRSA carriage $[13,14]$.

Healthcare workers (HCWs), situated at interface between the hospital and community, are an important reservoir of $S$. aureus for both HA-MRSA and CA-MRSA [15]. Colonized HCWs can transmit MRSA to patients, their families, and other HCWs and have been implicated as a source of transmission in outbreaks $[16,17]$. Identification of colonized HCWs combined with hand hygiene and other precautions have been shown to reduce the transmission and control the spread of MRSA [18].

Most studies of the transmission of MRSA have focused on isolates obtained from patients. Less is known about the frequency of carriage in HCWs, their genetic and clonal diversity, virulence gene determinants, and microbiological characteristics. The current study was designed to fill in some of these gaps.

The objectives were to determine the frequency of MRSA compared to methicillin-susceptible $S$. aureus (MSSA) nasal colonization in HCWs in relation to the intensity and duration of exposure to patients and characterize the molecular characteristics, antimicrobial resistant profiles, and biofilm-forming abilities of the isolates. The subjects included HCWs in an emergency department (ED), intensive care unit (ICU), and out-of-hospital emergency medical technicians (EMTs), and LTCFs.

\section{Methods}

\section{Study design}

This one-year prospective study was conducted from January to December 2015 at the National Cheng Kung University Hospital, Tainan, Taiwan, local fire department and long-term facilities. Healthcare providers who met the study criteria were offered the opportunity to participate in the study. The target population included out-of-hospital and in-hospital healthcare providers. Out-of-hospital providers were EMTs, student EMTs and staffs of LTCF, and in-hospital care providers included nurses and physicians working in the ED and ICU.

\section{Selection of participants}

Student EMTs, regarded as representatives of the general population with limited exposure to patients, were enrolled from the annual routine new EMT training program; paramedics and private ambulance EMTs with short term urgent care, transport to hospitals, or transfer of patients between healthcare facilities were selected from two local private ambulance groups. Physicians and nurses who worked in the adult medical ICU (MICU) and the medical ED (MED) were asked to participate. The participants must have had more than six months of working experience, willing to have a nasal swab and have not taken an antibiotic during the prior 21-days. They were asked to fill out an anonymous questionnaire regarding their place of work (current and previous), wearing adequate personal protective equipment and washing hands before and after patient care. 


\section{Microbiologic methods}

A sterile cotton swab was used to circle the anterior $1 \mathrm{~cm}$ of the nasal vestibule both nares. The swabs were immediately placed into transport medium (Venturi Transystem, Copan Innovation Ltd.) and brought to the microbiology laboratory. Swab samples were inoculated by the streak plate method on Trypticase soy agar with $5 \%$ sheep blood plates and incubated overnight at $37^{\circ} \mathrm{C}$. S. aureus was identified by colony morphology, gram stain and the coagulase test. MRSA were identified by the cefoxitin disk-diffusion method according to the recommendations of Clinical and Laboratory Standard Institutes [19].

\section{PFGE}

MRSA isolates were identified further by PFGE analysis with chromosomal DNA using the enzyme Smal. The relatedness of strains was determined by comparison of restriction fragment-length polymorphism in accordance with the guidelines published by Tenover et al. [20] PFGE patterns resulting in 2-3 band differences were considered to be closely related, those with 4-6 band differences were considered to be possibly related, and those with $\geq 7$ band differences were considered to be unrelated.

\section{Susceptibility testing}

The antimicrobial susceptibility of MRSA isolates to 10 antibiotics, including oxacillin, trimethoprim/ sulfamethoxazole, penicillin, teicoplanin, linezolid, clindamycin, doxycycline, fusidic acid, vancomycin, and erythromycin, was determined in accordance with the guideline of Clinical Laboratory Standards Institute [19].

\section{Biofilm formation assay}

Four microliters of a bacterial overnight culture were inoculated into $1 \mathrm{ml}$ of tryptic soy broth containing $0.25 \%$ glucose. An aliquot $(200 \mu \mathrm{l})$ of the sample was poured into each of a 96 -well polystyrene microplate (167008, Thermo Fisher Scientific, Waltham, MA, USA), and incubated for 3 days at $37^{\circ} \mathrm{C}$. The fluid was removed and the plate was stained with $0.1 \%$ safranin solution. The OD490 was measured using a microplate reader ( $\mu$ Quant, BioTek Instruments, Winooski, VT, USA).

\section{Molecular characterization}

Genomic DNA was obtained from the MRSA isolates by a Qiamp DNA mini kit protocol (Qiagen, Hilden, Germany) for molecular characterization. The presence of Panton-Valentine leucocidin ( $p v /$ gene and genes for fibronectin binding protein $A$ and $B(f n b A, f n b B)$ were determined by PCR as previous described $[21,22]$. The $S$. aureus MLST scheme uses internal fragments of the following seven house-keeping genes: arc, aro, glp, gmk, pta, tpi and yqi. PCR amplification was carried out on chromosomal DNA using an extension time of 30 seconds, and an annealing temperature of $55^{\circ} \mathrm{C}$, with Taq polymerase. The PCR products were then sequenced and the data were uploaded to the MLST website (http://www.mlst.net) for further analysis [23]. Typing of the staphylococcal chromosomal cassette mec ( $\mathrm{SCC} m e c$ ) was done by 
PCR with primers and by the methods published previously [24]. PCR for $\operatorname{mec} A$, mupA, and qacA/B were performed by the methods described previously $[23,25]$.

\section{Methods of measurements}

\section{Primary outcome}

Positive nasal swab for MSSA and MRSA were reported. Further nasal carriage of the MSSA and MRSA prevalence were calculated by descriptive statistics and cross tabulations to determine the frequency distribution of the MRSA nasal carriage among the different groups of healthcare professionals. Pearson's chi-square test, Fisher's exact test Cochran-Mantel-Haenszel test, logistic regression, and generalized linear models were used to compare MRSA colonization between groups. Odd ratios (ORs) were calculated with $95 \%$ confidence intervals (Cls). Student $t$ test or Mann-Whitney $U$ test were used to compared continuous variables. SAS software version 9.4 (SAS, Inc., Cary, North Carolina, USA) was applied for data entry, processing and statistical analysis.

\section{Secondary outcome analysis}

Positive MRSA samples were enrolled for further pathogenicity by molecular analysis. The drug susceptibility, basic molecular typing, virulence factors such as $p v /$ gene and genes for fibronectin binding protein and the biofilm formation assay were conducted.

\section{Results}

\section{Prevalence of MRSA and MSSA}

Two hundred and forty-eight healthcare providers were enrolled in the study. The distribution of the HCWs by site of work is shown in Figure 1. The frequency of isolation of MSSA and MRSA according to the characteristics of the study population is shown in Table 1.

S. aureus was isolated from 63 of the $248 \mathrm{HCWs} \mathrm{(25.4 \% ).} \mathrm{Fifteen} \mathrm{(23.8 \% )} \mathrm{of} \mathrm{isolates} \mathrm{were} \mathrm{MRSA.} \mathrm{The}$ overall MRSA nasal carriage rate was $6 \%(15 / 248)$. Most MRSA carriers were female $(14 / 15,93.3 \%)$, and HCWs who had worked in a facility for $\geq 5$ years $(11 / 15,73.3 \%)$. LCTFs had the highest prevalence $(3 / 25$, $12 \%)$, followed by hospital nurses $(11 / 103,10.7 \%)$ and EMTs $(1 / 62,1.6 \%)$. In contrast, the overall carriage of MSSA was $48 / 248$, (19.4\%); female (24/48, 50\%); and worked in a facility for $\geq 5$ years $(25 / 48,52.1 \%)$. Hospital nurses had the highest rate of MSSA nasal carriage $(22 / 103,21.4 \%)$ followed by EMTs $(12 / 62,19.4 \%)$ and LCTFs $(4 / 25,16 \%)$. None of 10 physicians and student EMTs was colonized by MRSA.

\section{Characterization of MRSA isolates}

All of the 15 MRSA isolates were positive for mecA by PCR. Most were SCCmec IV $(9,60 \%)$ and belonged to two pandemic CA-MRSA genotypes, ST59 $(6,67 \%)$ and ST45 (3, 33\%). All the isolates could be divided 
into 6 major clones by PFGE pattern analysis (Fig. 2). The most predominant pulsotype contained 5 isolates carrying SCCmec IV or V/PVL-/ST59. One isolate, belonging to ST398 and carrying SCCmec V, was isolated from a MICU nurse who had traveled to Europe within 12 months.

The MRSA isolates exhibited high rates of resistance to erythromycin (53\%) and clindamycin (53\%). Only one isolate was resistant to fusidic acid. One isolate from a MICU nurse was detected as qacA/B-positive, conferring resistance to chlorhexidine in S. aureus. The MIC to chlorhexidine was $4 \mathrm{mg} / \mathrm{L}$. None of the MRSA isolates were resistant by phenotypic or genetic tests to mupirocin, linezolid or glycopeptide antibiotics (vancomycin or teicoplanin).

All of the MRSA nasal colonizing isolates, regardless of the SCCmec type, formed low levels of biofilm that were indistinguishable from the CA-MRSA V/PVL+/ST59 clinical isolates (Fig. 3). In contrast, the traditional HA-MRSA III/PVL-/ST239 clinical isolates had significantly higher levels of biofilm formation. Additionally, all the MRSA nasal colonizing isolates had $f n b A$ gene but none possessed the $f n b B$ gene.

\section{Discussion}

We found that $25.4 \%$ of HCWs were nasal carriers of $S$. aureus. About a quarter (23.8\%) of the population was colonized by MRSA. The overall frequency of MRSA was $6 \%$. The prevalence of MRSA was highest among LTCF staffs, followed by ICU nurses, ED nurses and EMTs. SCCmec typing revealed that the majority of strains were type IV and V. These are the most common types of CA-MRSA in Taiwan.

These findings are consistent with the frequency of HCW MRSA nasal carriage in previous studies in Taiwan (5.0-7.8\%) [26], but higher than in other countries [15]. They are also consistent with other studies that found the highest prevalence of MRSA nasal carriage among HCWs with close contact with patients, poor attention to infection control policy, and high work-load [15]. Direct patient contact is considered to be the main transmission route for MRSA [27]. In the current study the greatest risk of MRSA nasal carriage was in HCWs working for 5 to 10 years.

Nearly all isolates in this study carried either SCCmec type IV or V, suggesting a community origin. Only one MRSA isolate with SCCmec type II, the traditional HA-MRSA type, was isolated from an ICU nurse. This finding indicates that the CA-MRSA types (type IV and V) were more common among healthcare providers and the HA-MRSA strain still remained in the hospital. This may be due to strict infection control measures in our hospital. Another possibility is clonal replacement in the hospital setting. In the past decade, CA-MRSA has been increasingly identified as a cause of hospital-onset and healthcare associated infections. This suggests that certain clones have the ability to cross the barriers between hospitals and the community $[28,29]$.

Several unusual MRSA strains were isolated from the current study population. An isolate from a nurse working in the MICU was SCCmec II ST5. This HA-MRSA strain carried chlorhexidine resistant genes $(q a c A / B)$. Chlorhexidine is widely used as antiseptic for central venous catheter care bundle and patient bathing to prevent nosocomial infection in out hospital. Chlorhexidine based soaps and mupirocin 
ointment are commonly used for cleaning and decontamination of MRSA. Prior investigators have also described MRSA strains carrying chlorhexidine and mupirocin resistant genes [30, 31], but these are uncommon in Taiwan.

Another unusual MRSA strain, ST398, was isolated from an MICU nurse. This is an important emerging strain associated with the livestock mainly in Europe and North America [32, 33]. MRSA ST398 is usually associated with pigs and veal calves but can colonize other host species. These include cows, sheep, poultry and farmers who are in frequent contact with MRSA-colonized animals, and can cause infections in humans [34]. The nurse who acquired this strain had traveled to Europe within 12 months and had contact to animals. The role of livestock as a potential source of MRSA infection is a growing public health problem. The risk and impact of HCWs carrying this clone need to be closely monitored.

Some MRSA strains are able to produce biofilm on both mucosal and inanimate surfaces, making them difficult to eradicate [35]. Biofilm formation is considered to have a role in $S$. aureus colonization [36, 37]. Recently fibronectin-binding proteins (Fnb A and Fnb B) have also been reported to play a role in biofilm formation. The association of the expression of $\mathrm{Fnb} A$ and $\mathrm{Fnb} B$ with increased bacterial aggregation suggests that fibronectin-binding proteins can promote the accumulation phase of biofilms [38]. In the current study all the MRSA nasal colonization isolates carried the fnbA but not the fnbB gene and only exhibited low levels of biofilm formation. This is consistent with the recent concept that a dispersed mode of growth in the vestibulum nasi is preferable to a biofilm mode during $S$. aureus nasal colonization [39].

We also found that MSSA strains were more abundant than MRSA in nasal carriers (19.4\%) and were differently distributed. In contrast to MRSA, MSSA exhibited an equal sex distribution and was much more likely to be isolated from EMTs. We believe this is important because all $S$. aureus have the potential to produce invasive disease and need to be included in surveillance studies and control measures.

This study has several limitations. First, it was conducted in a large metropolitan region in southern Taiwan and the findings may not be generalizable to other localities. Second, single cross-sectional sampling did not allow us to differentiate between transient and persistent carriers of MRSA and MSSA. Third, samples were collected only from the nares. It has been estimated that $15-50 \%$ of MRSA carriers are non-nasal [40]. Therefore it is likely that we underestimated the overall prevalence of MRSA and MSSA. Finally, whole-genome sequencing is more sensitive than molecular analysis to classify MRSA strains as community or hospital-associated. It has the added advantage of establishing genetic relatedness and recent transmission.

The strengths of this study include its prospective design, observation over a full year, adequate sample size of a diverse representative population of HCWs with major differences in their exposure to patients, comprehensive molecular characterization of the MRSA, biofilm-forming ability and antibiotic susceptibility testing.

\section{Conclusions}


This study demonstrates that nasal colonization by MRSA differs among healthcare professionals in relation to the extent and duration of exposure to patients. Most of the isolates belong to CA-MRSA strains, exhibited high rates of resistance to erythromycin and clindamycin, and produced low level of biofilms. An unusual MRSA strain, ST398, was isolated from an MICU nurse who had exposure to livestock in Europe. Another MICU nurse was colonized a qacA/B-positive strain conferring resistance to chlorhexidine. MRSA represents only the tip of the iceberg of nasal colonization by $S$. aureus. MSSA strains were 3.2 times more common and much more frequent in hospital nurses and EMTs who had limited exposure to patients. This supports the inclusion of all strains of $S$. aureus in surveillance and infection control programs.

\section{Abbreviations}

MRSA: Methicillin-resistant Staphylococcus aureus; CA-MRSA: community-associated Methicillinresistant Staphylococcus aureus; ED: emergency department; EMTs: emergency medical technicians; HAMRSA: healthcare-associated Methicillin-resistant Staphylococcus aureus; HCWs: Healthcare workers; ICU: intensive care unit; LTCF: long-term care facilities.

\section{Declarations}

\section{Acknowledgements}

We thank Dr. Calvin M. Kunin for providing invaluable suggestions and critical review of the manuscript.

\section{Funding}

This study is supported by the Research Centre of National Cheng Kung University Hospital (NCKUH10406035, NCKUH-10506016, and NCKUH-10605008). The funders played no role in study design, data collection and analysis, decision to publish, or preparation of the manuscript.

\section{Availability of data and materials}

Data and materials are available upon request to the corresponding author.

\section{Authors' contributions}

$\mathrm{HIS}, \mathrm{CYC}$ did the study design; HIS, $\mathrm{HCH}$ and $\mathrm{CHW}$ collected the study materials; HIS, HCH, FCS, YJL, CMC and $\mathrm{CYC}$ analyzed and interpreted the data; $\mathrm{HIS}, \mathrm{HCH}$ and $\mathrm{CYC}$ wrote the article.

\section{Ethics approval and consent to participate}

This study was conducted in accordance with the Helsinki Declaration. The study protocol and the study data were approved by the Institutional Review Board (IRB), National Cheng Kung University Hospital 
(BER-104-029). Written informed consent was obtained from each participant. Participants' information was kept anonymous and de-identified prior to the analysis.

\section{Consent for publication}

Not applicable.

\section{Conflicts of interest}

The authors declare that they have no competing interests.

\section{References}

1. Cosgrove SE, Sakoulas G, Perencevich EN, Schwaber MJ, Karchmer AW, Carmeli Y: Comparison of mortality associated with methicillin-resistant and methicillin-susceptible Staphylococcus aureus bacteremia: a meta-analysis. Clin Infect Dis. 2003;36(1):53-9.

2. Pallin DJ, Egan DJ, Pelletier AJ, Espinola JA, Hooper DC, Camargo CA, Jr.: Increased US emergency department visits for skin and soft tissue infections, and changes in antibiotic choices, during the emergence of community-associated methicillin-resistant Staphylococcus aureus. Ann Emerg Med. 2008;51(3):291-8.

3. DeLeo FR, Otto M, Kreiswirth BN, Chambers HF: Community-associated meticillin-resistant Staphylococcus aureus. Lancet. 2010;375(9725):1557-68.

4. Yamamoto T, Nishiyama A, Takano T, Yabe S, Higuchi W, Razvina O, Shi D: Community-acquired methicillin-resistant Staphylococcus aureus: community transmission, pathogenesis, and drug resistance. J Infect Chemother. 2010; 16(4):225-54.

5. Wertheim HF, Melles DC, Vos MC, van Leeuwen W, van Belkum A, Verbrugh HA, Nouwen JL: The role of nasal carriage in Staphylococcus aureus infections. Lancet Infect Dis. 2005;5(12):751-62.

6. Ellis MW, Hospenthal DR, Dooley DP, Gray PJ, Murray CK: Natural history of community-acquired methicillin-resistant Staphylococcus aureus colonization and infection in soldiers. Clin Infect Dis. 2004;39(7):971-9.

7. Perl TM, Cullen JJ, Wenzel RP, Zimmerman MB, Pfaller MA, Sheppard D, Twombley J, French PP, Herwaldt LA, Mupirocin et al: Intranasal mupirocin to prevent postoperative Staphylococcus aureus infections. N Engl J Med. 2002;346(24):1871-7.

8. van Rijen MM, Bonten M, Wenzel RP, Kluytmans JA: Intranasal mupirocin for reduction of Staphylococcus aureus infections in surgical patients with nasal carriage: a systematic review. J Antimicrob Chemother. 2008;61(2):254-61. 
9. Peters C, Dulon M, Kleinmuller O, Nienhaus A, Schablon A: MRSA Prevalence and Risk Factors among Health Personnel and Residents in Nursing Homes in Hamburg, Germany - A Cross-Sectional Study. PLoS One 2017;12(1):e0169425.

10. Gorwitz RJ, Kruszon-Moran D, McAllister SK, McQuillan G, McDougal LK, Fosheim GE, Jensen BJ, Killgore G, Tenover FC, Kuehnert MJ: Changes in the prevalence of nasal colonization with Staphylococcus aureus in the United States, 2001-2004. J Infect Dis. 2008;197(9):1226-34.

11. Huang YC, Su LH, Lin TY: Nasal carriage of methicillin-resistant Staphylococcus aureus among pediatricians in Taiwan. PLoS One. 2013;8(11):e82472.

12. Wang JT, Liao CH, Fang CT, Chie WC, Lai MS, Lauderdale TL, Lee WS, Huang JH, Chang SC: Prevalence of and risk factors for colonization by methicillin-resistant Staphylococcus aureus among adults in community settings in Taiwan. J Clin Microbiol. 2009;47(9):2957-63.

13. Mody L, Kauffman CA, Donabedian S, Zervos M, Bradley SF: Epidemiology of Staphylococcus aureus colonization in nursing home residents. Clin Infect Dis. 2008;46(9):1368-73.

14. Hidron Al, Kourbatova EV, Halvosa JS, Terrell BJ, McDougal LK, Tenover FC, Blumberg HM, King MD: Risk factors for colonization with methicillin-resistant Staphylococcus aureus (MRSA) in patients admitted to an urban hospital: emergence of community-associated MRSA nasal carriage. Clin Infect Dis. 2005;41(2):159-66.

15. Albrich WC, Harbarth S: Health-care workers: source, vector, or victim of MRSA? Lancet Infect Dis. 2008;8(5):289-301.

16. Eveillard M, Martin Y, Hidri N, Boussougant Y, Joly-Guillou ML: Carriage of methicillin-resistant Staphylococcus aureus among hospital employees: prevalence, duration, and transmission to households. Infect Control Hosp Epidemiol. 2004;25(2):114-20.

17. Harris SR, Cartwright EJ, Torok ME, Holden MT, Brown NM, Ogilvy-Stuart AL, Ellington MJ, Quail MA, Bentley SD, Parkhill J et al: Whole-genome sequencing for analysis of an outbreak of meticillin-resistant Staphylococcus aureus: a descriptive study. Lancet Infect Dis. 2013;13(2):130-6.

18. Guidelines for the Control of Methicillin-resistant Staphylococcus aureus in New Zealand [https://www.health.govt.nz/system/files/documents/publications/mrsa.pdf]

19. M100 Performance Standards for Antimicrobial Susceptibility Testing. In. Edited by Institute CaLS, 27th Edition edn: Clinical and Laboratory Standards Institute 2017.

20. Tenover FC, Arbeit RD, Goering RV, Mickelsen PA, Murray BE, Persing DH, Swaminathan B: Interpreting chromosomal DNA restriction patterns produced by pulsed-field gel electrophoresis: criteria for bacterial strain typing. J Clin Microbiol. 1995;33(9):2233-9. 
21. Lina G, Piemont Y, Godail-Gamot F, Bes M, Peter MO, Gauduchon V, Vandenesch F, Etienne J: Involvement of Panton-Valentine leukocidin-producing Staphylococcus aureus in primary skin infections and pneumonia. Clin Infect Dis. 1999;29(5):1128-32.

22. Mehrotra M, Wang G, Johnson WM: Multiplex PCR for detection of genes for Staphylococcus aureus enterotoxins, exfoliative toxins, toxic shock syndrome toxin 1 , and methicillin resistance. J Clin Microbiol. 2000;38(3):1032-5.

23. Enright MC, Day NP, Davies CE, Peacock SJ, Spratt BG: Multilocus sequence typing for characterization of methicillin-resistant and methicillin-susceptible clones of Staphylococcus aureus. J Clin Microbiol. 2000;38(3):1008-15.

24. Oliveira DC, de Lencastre H: Multiplex PCR strategy for rapid identification of structural types and variants of the mec element in methicillin-resistant Staphylococcus aureus. Antimicrob Agents Chemother. 2002;46(7):2155-61.

25. Mc Gann P, Milillo M, Kwak YI, Quintero R, Waterman PE, Lesho E: Rapid and simultaneous detection of the chlorhexidine and mupirocin resistance genes qacA/B and mupA in clinical isolates of methicillinresistant Staphylococcus aureus. Diagn Microbiol Infect Dis. 2013;77(3):270-2.

26. Huang YC, Chen CJ: Community-associated meticillin-resistant Staphylococcus aureus in children in Taiwan, 2000s. Int J Antimicrob Agents. 2011;38(1):2-8.

27. Weigelt JA, Lipsky BA, Tabak YP, Derby KG, Kim M, Gupta V: Surgical site infections: Causative pathogens and associated outcomes. Am J Infect Control. 2010;38(2):112-20.

28. O'Hara FP, Amrine-Madsen H, Mera RM, Brown ML, Close NM, Suaya JA, Acosta CJ: Molecular characterization of Staphylococcus aureus in the United States 2004-2008 reveals the rapid expansion of USA300 among inpatients and outpatients. Microb Drug Resist. 2012;18(6):555-61.

29. Chen CJ, Huang YC, Su LH, Wu TL, Huang SH, Chien CC, Chen PY, Lu MC, Ko WC: Molecular epidemiology and antimicrobial resistance of methicillin-resistant Staphylococcus aureus bloodstream isolates in Taiwan, 2010. PLoS One. 2014;9(6):e101184.

30. Sheng WH, Wang JT, Lauderdale TL, Weng CM, Chen D, Chang SC: Epidemiology and susceptibilities of methicillin-resistant Staphylococcus aureus in Taiwan: emphasis on chlorhexidine susceptibility. Diagn Microbiol Infect Dis. 2009;63(3):309-13.

31. Lee H, Lim H, Bae IK, Yong D, Jeong SH, Lee K, Chong Y: Coexistence of mupirocin and antiseptic resistance in methicillin-resistant Staphylococcus aureus isolates from Korea. Diagn Microbiol Infect Dis. 2013;75(3):308-12.

32. Smith TC, Male MJ, Harper AL, Kroeger JS, Tinkler GP, Moritz ED, Capuano AW, Herwaldt LA, Diekema DJ: Methicillin-resistant Staphylococcus aureus (MRSA) strain ST398 is present in midwestern U.S. swine 
and swine workers. PLoS One. 2009;4(1):e4258.

33. Wassenberg MW, Bootsma MC, Troelstra A, Kluytmans JA, Bonten MJ: Transmissibility of livestockassociated methicillin-resistant Staphylococcus aureus (ST398) in Dutch hospitals. Clin Microbiol Infect. 2011;17(2):316-9.

34. Garcia-Alvarez L, Holden MT, Lindsay H, Webb CR, Brown DF, Curran MD, Walpole E, Brooks K, Pickard DJ, Teale $C$ et al: Meticillin-resistant Staphylococcus aureus with a novel mecA homologue in human and bovine populations in the UK and Denmark: a descriptive study. Lancet Infect Dis. 2011;11(8):595-603.

35. Donlan RM, Costerton JW: Biofilms: survival mechanisms of clinically relevant microorganisms. Clin Microbiol Rev. 2002;15(2):167-93.

36. Iwase T, Uehara Y, Shinji H, Tajima A, Seo H, Takada K, Agata T, Mizunoe Y: Staphylococcus epidermidis Esp inhibits Staphylococcus aureus biofilm formation and nasal colonization. Nature. 2010;465(7296):346-9.

37. Quinn GA, Tarwater PM, Cole AM: Subversion of interleukin-1-mediated host defence by a nasal carrier strain of Staphylococcus aureus. Immunology. 2009;128(1 Suppl):e222-9.

38. McCourt J, O'Halloran DP, McCarthy H, O'Gara JP, Geoghegan JA: Fibronectin-binding proteins are required for biofilm formation by community-associated methicillin-resistant Staphylococcus aureus strain LAC. FEMS Microbiol Lett. 2014;353(2):157-64.

39. Krismer B, Peschel A: Does Staphylococcus aureus nasal colonization involve biofilm formation? Future Microbiol. 2011;6(5):489-93.

40. Lautenbach E, Nachamkin I, Hu B, Fishman NO, Tolomeo P, Prasad P, Bilker WB, Zaoutis TE: Surveillance cultures for detection of methicillin-resistant Staphylococcus aureus: diagnostic yield of anatomic sites and comparison of provider- and patient-collected samples. Infect Control Hosp Epidemiol. 2009;30(4):380-2.

\section{Tables}

For technical reasons the tables needed to be attached as a supplemental file and can be found below.

\section{Figures}




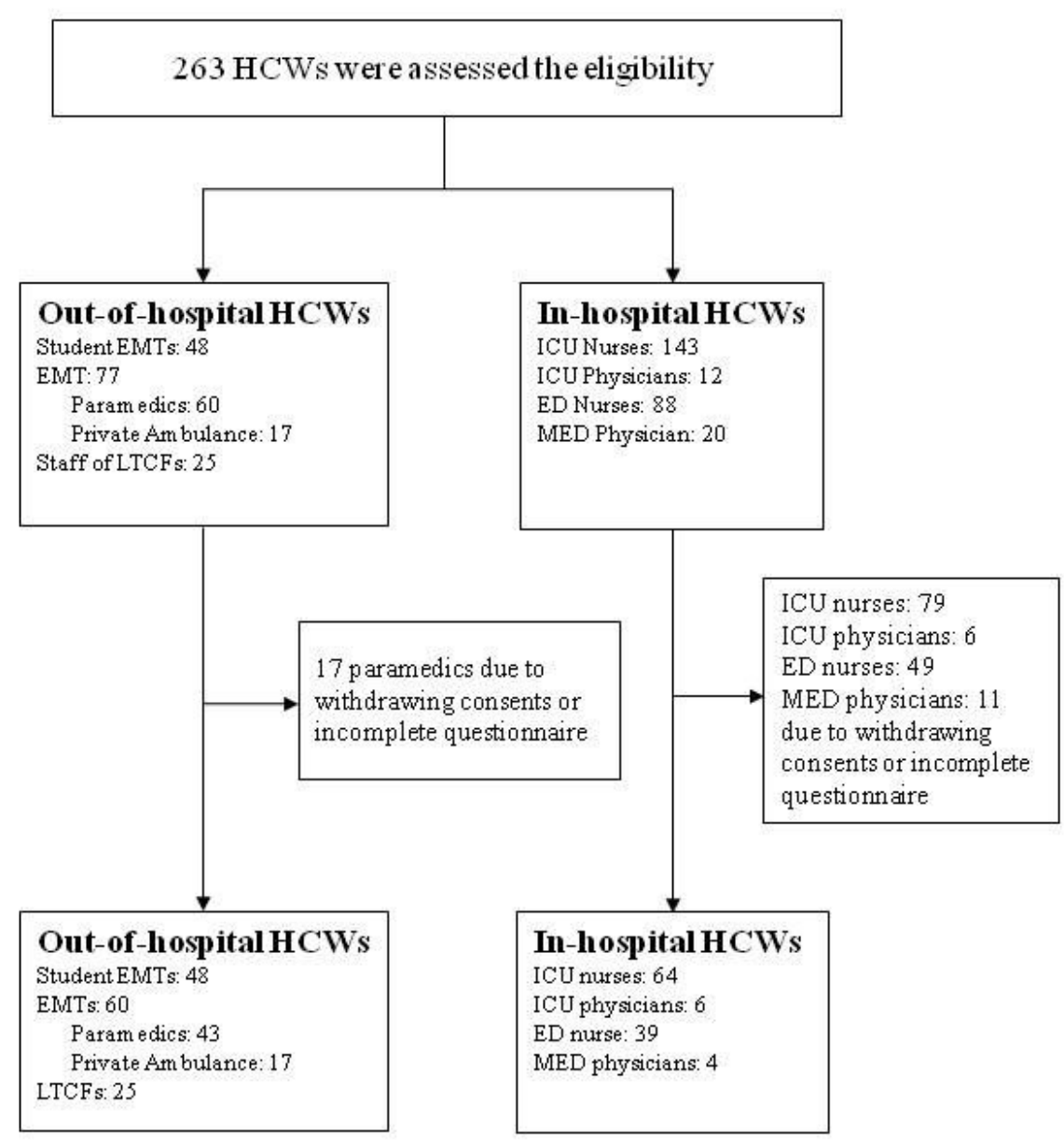

Figure 1

\section{Figure 1}

Enrollment of healthcare workers for MRSA and MSSA Nasal Carriage Study. ED, emergency department; EMT, emergency medical technician; ICU, intensive care unit; LTCF, long term care facility; MED, medical emergency department. 


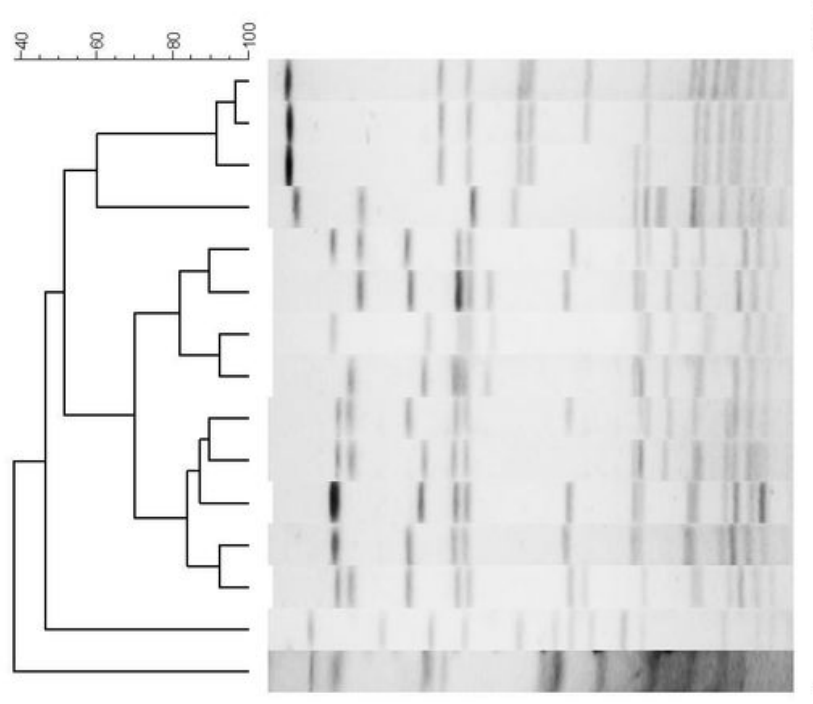

\begin{tabular}{|c|c|c|c|c|c|c|c|c|c|c|}
\hline Isolate ID & Ward/Staff & $\mathrm{scC} m e \mathrm{c}$ & MLST & $p v l$ & $f n b B$ & $q a c A / B$ & ERY & $\mathrm{CC}$ & SXT & $\mathrm{FA}$ \\
\hline 162 & EMTP & IV & 45 & - & - & - & $R$ & s & $s$ & $s$ \\
\hline 210 & SICU/N & IV & 45 & - & - & - & s & $s$ & $s$ & $s$ \\
\hline 177 & MICU/N & IV & 45 & - & - & - & s & s & s & $s$ \\
\hline 146 & LTCF/N & v & 45 & - & - & - & s & s & s & $R$ \\
\hline 5 & $E D / N$ & IV & 59 & - & - & - & $R$ & $R$ & s & s \\
\hline 80 & $E D / N$ & IV & 59 & + & - & - & $R$ & $R$ & s & s \\
\hline 1 & $E D / N$ & v & 59 & + & - & - & s & s & s & s \\
\hline 193 & LTCF/SW & v & 59 & + & - & - & s & s & s & s \\
\hline 194 & LTCF/N & IV & 59 & - & - & - & $R$ & $R$ & s & $s$ \\
\hline 198 & MICU/N & v & 59 & - & - & - & s & s & s & $s$ \\
\hline 172 & MICU/N & IV & 59 & - & - & - & s & s & s & s \\
\hline 235 & $\mathrm{SICU} / \mathrm{N}$ & IV & 59 & - & - & - & $R$ & $R$ & s & $s$ \\
\hline 78 & $E D / N$ & IV & 59 & - & - & - & $R$ & $R$ & s & S \\
\hline 168 & MICU/N & ॥ & 5 & - & - & + & $R$ & $R$ & $s$ & s \\
\hline 164 & MICU/N & v & 398 & - & - & - & $R$ & 1 & $s$ & s \\
\hline
\end{tabular}

\section{Figure 2}

Molecular characterization, antibiogram of nasal carriage isolates and the PFGE dendrogram compares fingerprint patterns of the methicillin-resistant Staphylococcus aureus (MRSA) isolates from 15 healthcare workers. SCCmec and MLST indicate the results for MRSA type. Columns marked "pvl", "fnbB", and qacA/B" are the results for genetic tests performed to detect the PVL, fibronectin $B$ and chlorhexidine resistance genes. ERY, erythromycin; CC, clindamycin; SXT, trimethoprim-sufamethoxazole; FA, fucidic acid; S, susceptible; R, resistant. ED, Emergency department; EMTP, Emergency Medical Technician Professional (Paramedic); MICU, Medical ICU; N, nurse; SW, Social Worker; SICU, Surgical ICU; LTCF, Long term care facility. 


\section{Figure 3}

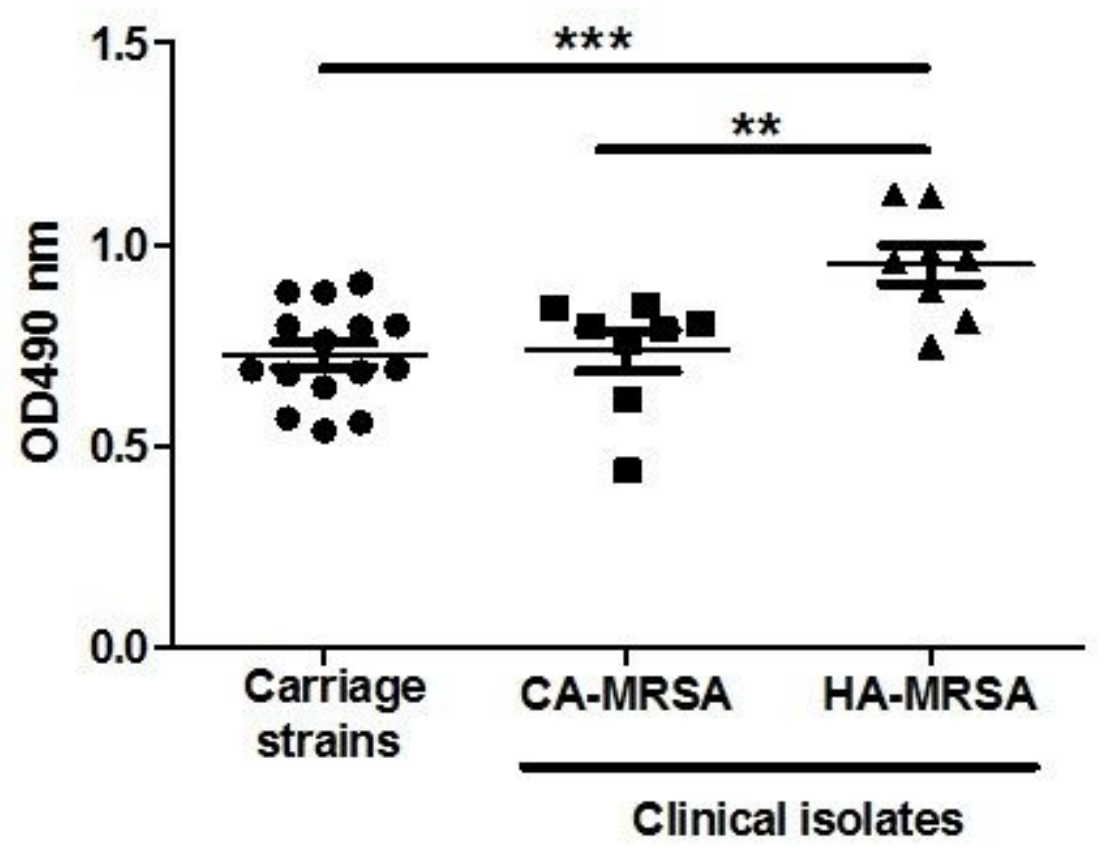

Figure 3

Biofilm formation ability. Biofilm formation of the MRSA nasal colonization isolates (carriage), CA-MRSA V/PVL+/ST59 clinical isolates (CA-MRSA), and the HA-MRSA III/PVL-/ST239 (HA-MRSA) onto

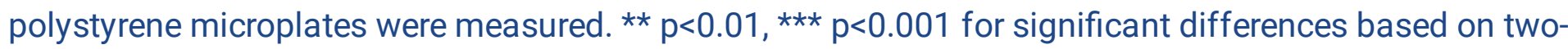
sided unpaired t test.

\section{Supplementary Files}

This is a list of supplementary files associated with this preprint. Click to download.

- supplement1.docx 